\title{
Computational Pathology Analysis of Tissue Microarrays Predicts Survival of Renal Clear Cell Carcinoma Patients
}

\author{
Thomas J. Fuchs ${ }^{1,3}$, Peter J. Wild ${ }^{2}$, \\ Holger Moch ${ }^{2,3}$, and Joachim M. Buhmann ${ }^{1,3}$ \\ ${ }^{1}$ Institute for Computational Science, ETH Zürich, Switzerland \\ \{thomas.fuchs, jbuhmann\}@inf.ethz.ch \\ ${ }^{2}$ Institute of Pathology, University Hospital Zürich, University Zürich, Switzerland \\ ${ }^{3}$ Competence Center for Systems Physiology and Metabolic Diseases, ETH Zürich
}

\begin{abstract}
Renal cell carcinoma (RCC) can be diagnosed by histological tissue analysis where exact counts of cancerous cell nuclei are required. We propose a completely automated image analysis pipeline to predict the survival of RCC patients based on the analysis of immunohistochemical staining of MIB-1 on tissue microarrays. A random forest classifier detects cell nuclei of cancerous cells and predicts their staining. The classifier training is achieved by expert annotations of 2300 nuclei gathered from tissues of 9 different RCC patients. The application to a test set of 133 patients clearly demonstrates that our computational pathology analysis matches the prognostic performance of expert pathologists.
\end{abstract}

\section{Introduction}

Tissue Microarray Analysis (TMA): We propose a computational pathology framework that is tailored to renal cell carcinoma (RCC) which is one of the ten most frequent malignancies in Western societies. The identification of biomarkers for prediction of prognosis (prognostic marker) or response to therapy (predictive marker) is of utmost importance. The tissue microarray technology (Fig. 1) promises to significantly accelerate studies seeking for associations between molecular changes and clinical endpoints 1. Sections from such array blocks can be used for simultaneous in situ analysis (DNA, RNA, protein) of up to thousand primary tumors.

Although the production of tissue microarrays is an almost routine task for most laboratories, the evaluation of stained tissue microarray slides remains tedious, time consuming and prone to error. Current image analysis software requires extensive user interaction to properly identify cell populations, to select regions of interest for scoring, to optimize analysis parameters, and to organize the resulting raw data. Because of these drawbacks in current software, typically pathologists manually assign a composite staining score for each spot during multiple microscopy sessions over a period of days. Manual scoring also introduces a major bottleneck that hinders the use of tissue microarrays in high-throughput 
analysis of multiple markers. The workflow in manual TMA analysis is as follows: (i) spots on the slide are detected, (ii) the cancerous nuclei are identified, and (iii) the percentage of immunohistochemically stained cancerous nuclei is estimated.

In the domain of cytology, especially blood analysis and smears, automated analysis has been already established 2. The big difference to histological tissues is the homogeneous background on which the cells are clearly distinguishable and the absence of vessels and connective tissue. The isolation of cells simplifies the detection and segmentation process of the cells significantly. A similar simplification can be seen in the field of immunofluorescence imaging. Although studies were conducted on a global estimation of staining on TMA spots 34 and on classifying whole spots [5], to our knowledge this is the first in depth study for tissue microarrays which incorporates expert labeling information down to the detail and precision of single cell nuclei.

Motivation: The absence of an objective ground truth requires us to generate a gold standard by combining the knowledge of expert pathologists. These labels are indispensable for the training of classifiers, for their validation and it is highly non-trivial to acquire them from a technical as well as statistical viewpoint.

To facilitate the labeling process for trained pathologists, we developed dedicated labeling tools for TMA spots. The software enables the user to view single TMA spots and it provides zooming and scrolling capabilities. It is possible to annotate the image with vectorial data in SVG (support vector graphics) format and to mark cell nuclei, vessels and other biological structures. An additional demand to the software was the usability on a tablet PC so that a pathologist can perform all operations with a pen alone in a simple an efficient manner. We set up three labeling experiments:

1. The first experiment was conducted to investigate the inter pathologist variability for estimating nuclear staining. Therefore 14 trained pathologists from the University Hospital Zürich estimated MIB-1 staining on nine TMA spots. The results show a large disagreement of the pathologist on spots with an averages staining of more than $10 \%$. On one spot, for example, the standard deviation was more than $20 \%$. This result motivates and emphasizes the need for an objective estimation algorithm.

2. In the second experiment, two pathologists and experts in renal cell carcinoma marked the location of each cell nucleus and its approximate size on the same nine spots. In total each pathologist has detected more than 2300 cell nuclei on these images. This tedious process demanded several days of work and was performed independently of each other. These labels are used to extract training examples for the nuclei detection algorithm described in Section 2]

3. The third experiment was designed on one hand to learn the difference between cancerous and benign nuclei and on the other hand to learn the difference between stained and non-stained nuclei. Therefore, five trained pathologists classified 180 nuclei randomly drawn from the set of nuclei detected in the second 


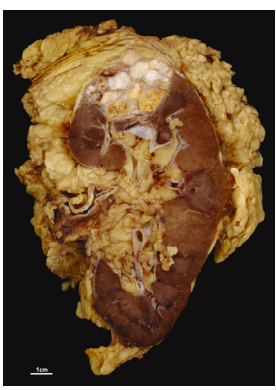

(a)

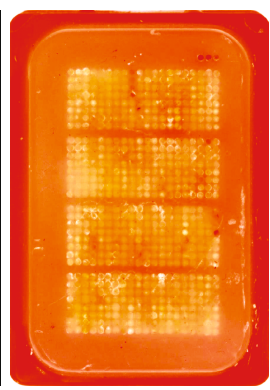

(b)

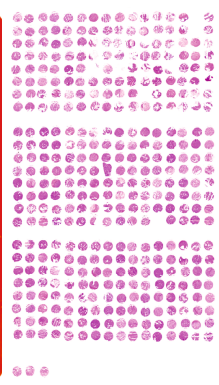

(c)

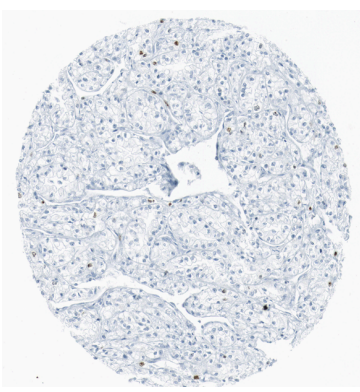

(d)

Fig. 1. Tissue Microarray Analysis (TMA): Primary tissue samples are taken from a cancerous kidney (a). Then $0.6 \mathrm{~mm}$ tissue cylinders are punched from the primary tumor block of different patients and arrayed in a recipient paraffin block (b). Slices of $0.6 \mu \mathrm{m}$ are cut off the paraffin block and are immunohistochemically stained (c). These slices are scanned and each spot represents a different patient. Image (d) depicts the TMA spot from a single patient with clear cell renal cell carcinoma stained with the MIB-1 (Ki-67) antigen.

experiment. In addition they estimated the immunohistochemical staining for the subset of nuclei which they classified as tumor.

The main challenge in this setting is posed by the vast heterogeneity of nuclei. In the domain of face detection similar techniques are used, but the inner class variability is significantly lower than for cell nuclei. The frontal faces can easily be centered and scaled and it has been shown [6] that Haar features represent the characteristics of a face quite well by taking advantage of e.g. the brighter ridge of the nose between the darker regions of the eye. In the domain of cell nuclei detection we do not have such a standardized preprocessing. The difficulties mainly arise from the following peculiarities of histopathological image processing: (i) The objects which can be found are not only benign or malignant renal nuclei, but the tissue also comprises endothelial cells, lymphocytes, erythrocytes etc.. Endothelial cells for example are extremely elongated where on the other hand lymphocytes are nearly perfectly round and homogeneous. Neither of these cells must be counted in the estimation process. (ii) A grave difficulty is the fact that nuclei as three dimensional structures are not always perfectly cut in their maximum dimension producing numerous cutting artifacts. For many of these artifacts it is not possible to determine if they are atypical or benign. (iii) Variations in the production process of TMAs can lead to areas of different thickness within one section. This preprocessing artifact produces blurred regions in the image during the scanning process.

Tissue Preparation and Scanning: The tissue microarray block was generated in a trial from the University Hospital Zürich. The TMA slides were immunohistochemically stained with the MIB-1 (Ki-67) antigen and scanned on a Nanozoomer C9600 virtual slide light microscope scanner from HAMAMATSU 
Photonics K.K.. The magnification of 40x resulted in a per pixel resolution of $0.23 \mu \mathrm{m}$. Finally the spots of single patients were extracted as separate three channel color images of $3000 \times 3000$ pixels size.

Problem Formulation: We formulate the problem of delineating the cell nuclei from the background and other structures as object detection task. In general two frameworks are possible. One could mimic the workflow of the pathologists, by first detecting all cell nuclei followed by classifying the nuclei into cancerous and benign before estimating the staining on the subset of cancerous nuclei. The second approach, which we follow, combines the detection and classification into one step. Therefore we train a classifier which only detects cancerous nuclei while ignoring erythrocytes, lymphocytes and nuclei from endothelial cells. We then calculate the percentage of stained nuclei from all detected nuclei. The final pipeline consist of the following stages: (i) Nuclei Detection, (ii) Staining Estimation, (iii) Survival Prediction.

Training Data: The training data consists in total of 2382 manually detected cell nuclei form nine different spots. For each of these nuclei a trained pathologist marked the center and the approximate radius of the nucleus. Based on the results and the exemplars from the classification labeling experiment 202 cell nuclei out of the 2382 where selected as positive training examples. This set was increased to 1212 by rotating and flipping the original patches as well as the transposed patches. In addition 1291 negative examples where collected which do not contain a nucleus but background and connective tissue. All nuclei were scaled to a radius of 15 pixels and patches of $65 \times 65$ pixels size were extracted. Hence the patches contained a lot of surrounding area compared to the nuclei with a diameter of 30 pixels. In contrast to face detection the surrounding of the objects is crucial for the classification. Nuclei often do not differ from connective tissue by their color or texture but only by their shape and their surrounding. Cancerous nuclei for example often show a bright corona around the nucleus membrane.

\section{Methods}

LBP and Color Feature Extraction: Local Binary Patterns (LBP) and color features are employed to build a histogram for the description of each image patch. LBP have the great advantage that they are illumination invariant, i.e. invariant with respect to monotonic grey scale-changes and therefore no grey-scale normalization or histogram equalization is needed. This reduces the runtime for feature extraction significantly. In face recognition excellent results were achieved by using the Local Binary Patterns (LBP) method. Ahonen et al. 7] have shown that regarding the recognition accuracy LBP outperform all present high performance methods including Principal Component Analysis (PCA), Linear Discriminant Analysis (LDA), Bayesian extra/intra personal classifiers and Elastic Bunch Graph Matching (EBGM). In addition LBP were also successfully applied to face detection 8]. To incorporate color information each 
of the three channels is resized to a $5 \times 5$ pixels image and the concatenation of these vectors is added to the final histogram. Finally each patch is described by a histogram of 331 bins, consisting of 75 color features and the LBP histogram of length 256.

Object Detection with Random Forests: Random forests 9 are employed to learn a classifier for the detection of cell nuclei. A random forest classifier consists of a collection of tree-structured classifiers $\left\{h\left(\mathbf{x}, \Theta_{k}\right), k=1, \ldots\right\}$ where $\left\{\Theta_{k}\right\}$ are independent identically distributed random vectors and each tree casts a unit vote for the most popular class at input $\mathbf{x}$.

Boosting classifiers in conjunction with a decision cascade became the de facto standard in object detection since Viola and Jones [6] published their highly influential paper on face detection. As a result today there exist a vast number of variations and extensions of their algorithm. Despite that we selected random forests for classification for the following reasons: (i) it has been shown that random forests outperform AdaBoost [10] regarding the generalization error while at the same time are more robust to noise [9. Especially this robustness is an important property taking into account the much higher variation in the data compared to other scenarios as described in section 1 . (ii) Trees are able to capture more complicated structures and relations between features than simple decision stumps as used in [6] and most Boosting classifiers. (iii) The internal out of bag (OOB) error provides an unbiased estimate of the generalization error in a framework were cross validation is not feasible due to excessively long training times of several weeks [6]. (iv) The clinical scenario does not demand a real time system. Therefore it is not necessary to split the classifier into a cascade and hence loosing the advantages of (i) to (iii). To our knowledge, random forests are applied for the first time to object detection in this work, while they have been recently used for image classification [11.

We have trained a random forest by randomly selecting 20 variables at each node as candidates for the split. This is in line with the suggestion of Breiman [9] who reports near optimum results for the square root of the number of features. We learn a forest up to 500 trees and protocol the estimated generalization error. The classifier converges relatively fast after approximately 80 trees to a minimal classification error of approximately $2 \%$. Due to performance considerations we finally used a forest with only 30 trees. This final classifier shows a slightly higher error of $3 \%$ but it executes significantly faster due to the small number of trees.

For cell nuclei detection we classify each patch of size $65 \times 65$ on a grid with $\Delta=3$. Therefore, 100,000 positions are evaluated for each spot of size $3000 \times 3000$ pixels. The classification results is stored in an accumulator image with a probability at each pixel for being a cell nucleus or not. After non maxima suppression the detections within a range of 20 pixels were clustered to one final hit which is approximately the size of a small nucleus. To assess the validity of such a hit the sum of its $15 \times 15$ surrounding was used as score. The threshold on this score is the only tuning parameter of the model. Such a parameter which is set to 10 in our experiments is unavoidable for any algorithm which has to evaluate accumulator images. 
To differentiate between stained and non-stained nuclei we train a single decision tree with the color features described above. This tree degrades to a single split on the center pixel of the blue channel on the training patches. Due to the fact, that the detection of the random forest is not fully centered on a nucleus we extend this notion and classify a nucleus as stained if one of the $3 \mathrm{x} 3$ central color features in the blue channel is below 0.64 .

Survival Analysis: The only objective and undisputed target in the medical domain relates to the survival of the patient. The experiments described in Section 1 show the large disagreement between pathologists for the estimation of staining. Therefore, the adaption of an algorithm to the estimates of one pathologist or to a consensus voting of a cohort of pathologist is not desirable. Hence we validate our algorithm against the right censored clinical survival data of 133 patients. In addition these results were compared to the estimations of an expert pathologist specialized on renal cell carcinoma. He analyzed all spots in an exceptional thorough manner which required him more than two hours. This time consuming annotation exceeds the standard clinical practice significantly by a factor of 10-20 and, therefore the results can be viewed as an excellent human estimate for this dataset. The main goal of tissue microarray analysis for the proliferation marker MIB-1 is the search for subgroups of patients which show different survival outcomes. Therefore, the patients are split in two $(1 / 2: 1 / 2)$ or three $(1 / 3: 1 / 3: 1 / 3)$ groups based on the estimated percentage of cancerous nuclei which express MIB-1. Then the Kaplan-Meier estimator is calculated for each subgroup. This involves first ordering the survival times from the smallest to the largest such that $t_{1} \leq t_{2} \leq t_{3} \leq \ldots \leq t_{n}$, where $t_{j}$ is the $j$ th largest unique survival time. The Kaplan-Meier estimate of the survival function is then obtained as

$$
\hat{S}(t)=\prod_{j: t_{(j)} \leq t}\left(1-\frac{d_{j}}{r_{j}}\right)
$$

where $r_{j}$ is the number of individuals at risk just before $t_{j}$, and $d_{j}$ is the number of individuals who die at time $t_{j}$.

To measure the goodness of separation between two or more groups, the logrank test (Mantel-Haenszel test) is employed which assesses the null hypothesis that there is no difference in the survival experience of the individuals in the different groups. The test statistic of the log-rank test (LRT) is $\chi^{2}$ distributed: $\hat{\chi^{2}}=\left[\sum_{i=1}^{m}\left(d_{1 i}-\hat{e}_{1 i}\right)\right]^{2} / \sum_{d_{i}=1}^{m} \hat{v}_{1 i}$ where $d_{1 i}$ is the number of deaths in the first group at $t_{i}$ and $e_{1 i}=n_{1 j} \frac{d_{i}}{n_{i}}$ where $d_{i}$ is the total number of deaths at time $t_{(i)}$, $n_{j}$ is the total number of individuals at risk at this time, and $n_{1 i}$ the number of individuals at risk in the first group.

Furthermore we use Cox's proportional hazard model to perform a univariate Cox regression on the survival target. This procedure models the hazard function $h(t)$ on the estimated percentage of stained nuclei without requiring restrictive assumptions about the dependence of the hazard function on time. The univariate model $\ln h(t)=\ln \alpha(t)+\beta x$ depends on the estimated percentage $x$ and $\alpha(t)$ denotes the baseline hazard function. The parameter $\beta$ is obtained by 

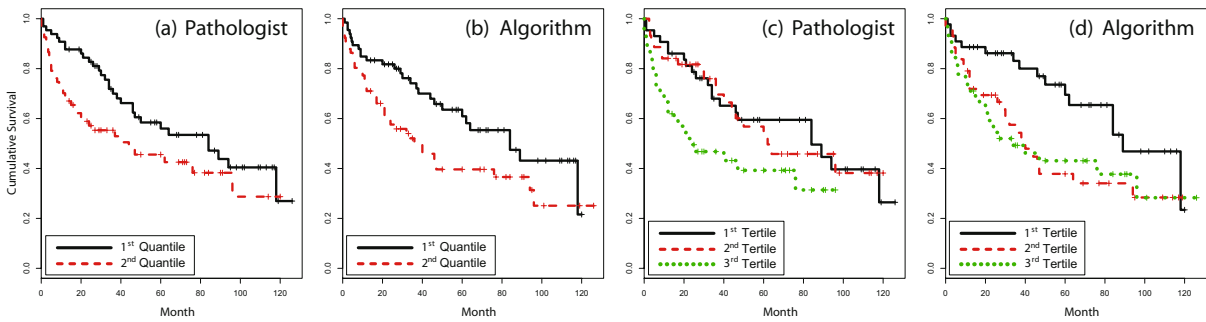

Fig. 2. Kaplan-Meier estimators showing significantly different survival times for renal cell carcinoma patients with high and low proliferating tumors. Compared to the manual estimation from the pathologist (a), the fully automatic estimation from the algorithm (b) performs equally well in terms of survival prediction on the partitioning of patients into two groups of equal size. Due to the higher resolution of the automatic estimation for tissues with low proliferation, the system performs better than the human expert for the identification of patients with very optimistic outcome (black curve in plot $(\mathrm{d}))$. In contrast the pathologist seems to perform better on selecting patients with very pessimistic outcome (dotted line in plot (c)).

maximizing the partial log likelihood and it depends only on the order in which events occur, not on the exact times of their occurrence. As is common practice, we report the p-value of the Wald-Statistic for Cox regression.

\section{Results}

The test set consisted of MIB-1 stained tissue microarray spots of 133 patients. We processed all spots with the random forest detection model on a high performance 64Bit cluster. The calculations were run in parallel on 100 cores and the analysis of one spot took approximately 4.2 hours. On average each spot contained 809 cell nuclei with a standard deviation of 391. In total the algorithm detected 107, 572 cell nuclei on all 133 spots.

When comparing the distributions of the estimated percentage of stained nuclei between the pathologist and our algorithm it can be seen that the distribution of the pathologist drastically peeks around $1.5 \%$ where the distribution of the algorithm is much smoother over the whole spectrum. Again it has to be noted that this shows the estimate of one pathologist and that the variability is expected to be vast, as shown in Section [1. The smoother distribution of the algorithm for the estimate leads to a finer resolution in low stained tissue.

We investigated the predictive power on a partitioning into two groups of equal number of patients and on a partitioning into three groups of equal size. The final results are depicted in Figure 2.

A significant difference in survival times can be seen for renal cell carcinoma patients with high and low proliferating tumors. This shows that MIB-1 staining is a univariate prognostic factor for the survival probability of renal cell 
carcinoma patients. The manual estimation from the pathologist can differentiate between patients of high and low risk with a LRT p-value of 0.043 . The automatic estimation from the algorithm performs equally well with a p-value of 0.026 . In addition we calculated the Wald-Test of the Cox regression which reports significant values for the pathologist $(p=0.045)$ and for our algorithm $(p=0.027)$.

\section{Conclusion}

Our computational pathology system objectively and automatically estimates a prognostic marker. This leads to a survival prediction of renal cell cancer patients with the same or superior accuracy as trained medical experts. To the best of our knowledge this is the first framework which is able to achieve this goal. Furthermore our algorithm shows higher precision with a low degree of staining, resulting in a more reliable identification of patients with excellent prognosis. The computational pathology system supports a rigorous sensitivity analysis of the medical decision process in RCC diagnosis and it promises further medical discoveries by histopathological tissue analysis.

\section{References}

1. Kononen, J., Bubendorf, L., et al.: Tissue microarrays for high-throughput molecular profiling of tumor specimens. Nat. Med. 4(7), 844-847 (1998)

2. Yang, L., Meer, P., Foran, D.J.: Unsupervised segmentation based on robust estimation and color active contour models. IEEE Transactions on Information Technology in Biomedicine 9(3), 475-486 (2005)

3. Yang, L., Chen, W., Meer, P., Salaru, G., Feldman, M.D., Foran, D.J.: High throughput analysis of breast cancer specimens on the grid. Med. Image Comput. 10 (Pt 1), 617-625 (2007)

4. Rabinovich, A., Agarwal, S., Laris, C.A., Price, J., Belongie, S.: Unsupervised color decomposition of histologically stained tissue samples

5. Hall, B., Chen, W., Reiss, M., Foran, D.J.: A clinically motivated 2-fold framework for quantifying and classifying immunohistochemically stained specimens, pp. 287294 (2007)

6. Viola, P., Jones, M.: Rapid object detection using a boosted cascade of simple features (2001)

7. Ahonen, T., Hadid, A., Pietikainen, M.: Face recognition with local binary patterns. In: Pajdla, T., Matas, J(G.) (eds.) ECCV 2004. LNCS, vol. 3021, pp. 469-481. Springer, Heidelberg (2004)

8. Hadid, A., Pietikainen, M., Ahonen, T.: A discriminative feature space for detecting and recognizing faces. In: CVPR 2004, June 27- July 2, 2004, vol. 2, II-797-II-804 (2004)

9. Breiman, L.: Random forests (1999)

10. Freund, Y., Schapire, R.: Experiments with a new boosting algorithm. In: Machine Learning: Proceedings of the Thirteenth International Conference, pp. 148-156 (1996)

11. Bosch, A., Zisserman, A., Munoz, X.: Image classification using random forests and ferns. In: ICCV 2007, October 14-21, 2007, pp. 1-8 (2007) 